\title{
Corrigendum: Multi-modal Brain MRI in Subjects with PD and iRBD
}

\author{
Silvia Mangia ${ }^{1 *}$, Alena Svatkova ${ }^{2,3}$, Daniele Mascali ${ }^{4}$, Mikko J. Nissi $^{5}$, Philip C. Burton ${ }^{1}$, \\ Petr Bednarik ${ }^{1,3}$, Edward J. Auerbach ${ }^{1}$, Federico Giove ${ }^{4,6}$, Lynn E. Eberly ${ }^{7}$, \\ Michael J. Howell ${ }^{8}$, Igor Nestrasil ${ }^{2}$, Paul J. Tuite ${ }^{8}$ and Shalom Michaeli ${ }^{1}$ \\ ${ }^{1}$ Department of Radiology, Center for Magnetic Resonance Research (CMRR), University of Minnesota, Minneapolis, MN, \\ United States, ${ }^{2}$ Department of Pediatrics, University of Minnesota, Minneapolis, MN, United States, ${ }^{3}$ Central European \\ Institute of Technology (CEITEC), Masaryk University, Brno, Czechia, ${ }^{4}$ MARBILab, Centro Fermi - Museo Storico Della Fisica \\ e Centro di Studi e Ricerche Enrico Fermi, Rome, Italy, ${ }^{5}$ Department of Applied Physics, University of Eastern Finland, \\ Kuopio, Finland, ${ }^{6}$ Fondazione Santa Lucia IRCCS, Rome, Italy, ${ }^{7}$ Division of Biostatistics, University of Minnesota, \\ Minneapolis, MN, United States, ${ }^{8}$ Department of Neurology, University of Minnesota, Minneapolis, MN, United States
}

Keywords: rotating frame MRI, Parkinson's disease, iRBD, functional connectivity, DTI

\section{OPEN ACCESS}

Edited and reviewed by: Kevin J. Black,

Washington University in St. Louis,

United States

*Correspondence:

Silvia Mangia

mangia@umn.edu

Specialty section:

This article was submitted to

Brain Imaging Methods,

a section of the journal

Frontiers in Neuroscience

Received: 30 April 2018

Accepted: 12 June 2018

Published: 26 June 2018

Citation:

Mangia S, Svatkova A, Mascali D, Nissi MJ, Burton PC, Bednarik P,

Auerbach EJ, Giove F, Eberly LE, Howell MJ, Nestrasil I, Tuite PJ and

Michaeli S (2018) Corrigendum:

Multi-modal Brain MRI in Subjects with $P D$ and $i R B D$.

Front. Neurosci. 12:446. doi: 10.3389/fnins.2018.00446

\section{A corrigendum on}

\section{Multi-modal Brain MRI in Subjects with PD and iRBD}

by Mangia, S., Svatkova, A., Mascali, D., Nissi, M. J., Burton, P. C., Bednarik, P., et al. (2017). Front. Neurosci. 11:709. doi: 10.3389/fnins.2017.00709

In the original paper there were errors in Figure $\mathbf{4}$ and in the text at page 7 . Figure $\mathbf{4}$ erroneously reported four asterisks, namely on top of T $1 \rho$ of putamen, T $2 \rho$ of midbrain, RAFF 4 of midbrain, and ReHo of amygdala. The figure also did not report one asterisk on top of T1 $\rho$ of SNc. The correct version of Figure 4 appears below.

In addition, the text at page 7 "Group differences between PD and controls were also observed for $\mathrm{T}_{2 \rho}$ in the amygdala $(p=0.033)$ and thalamus $(p=0.08)$ " should read as "Group differences between PD and controls were also observed for $\mathrm{T}_{2 \rho}$ in the amygdala $(p=0.033)$ and thalamus $(p$ $=0.008)$ ".

The authors sincerely apologize for these errors that may cause confusion for the reader. These errors however do not change the interpretation of the results and the main message of the study in any way, because the interpretation was based on the correct results reported in the Supplementary Table.

The original article has been updated.

Conflict of Interest Statement: The authors declare that the research was conducted in the absence of any commercial or financial relationships that could be construed as a potential conflict of interest.

Copyright ( 2018 Mangia, Svatkova, Mascali, Nissi, Burton, Bednarik, Auerbach, Giove, Eberly, Howell, Nestrasil, Tuite and Michaeli. This is an open-access article distributed under the terms of the Creative Commons Attribution License (CC BY). The use, distribution or reproduction in other forums is permitted, provided the original author(s) and the copyright owner are credited and that the original publication in this journal is cited, in accordance with accepted academic practice. No use, distribution or reproduction is permitted which does not comply with these terms. 


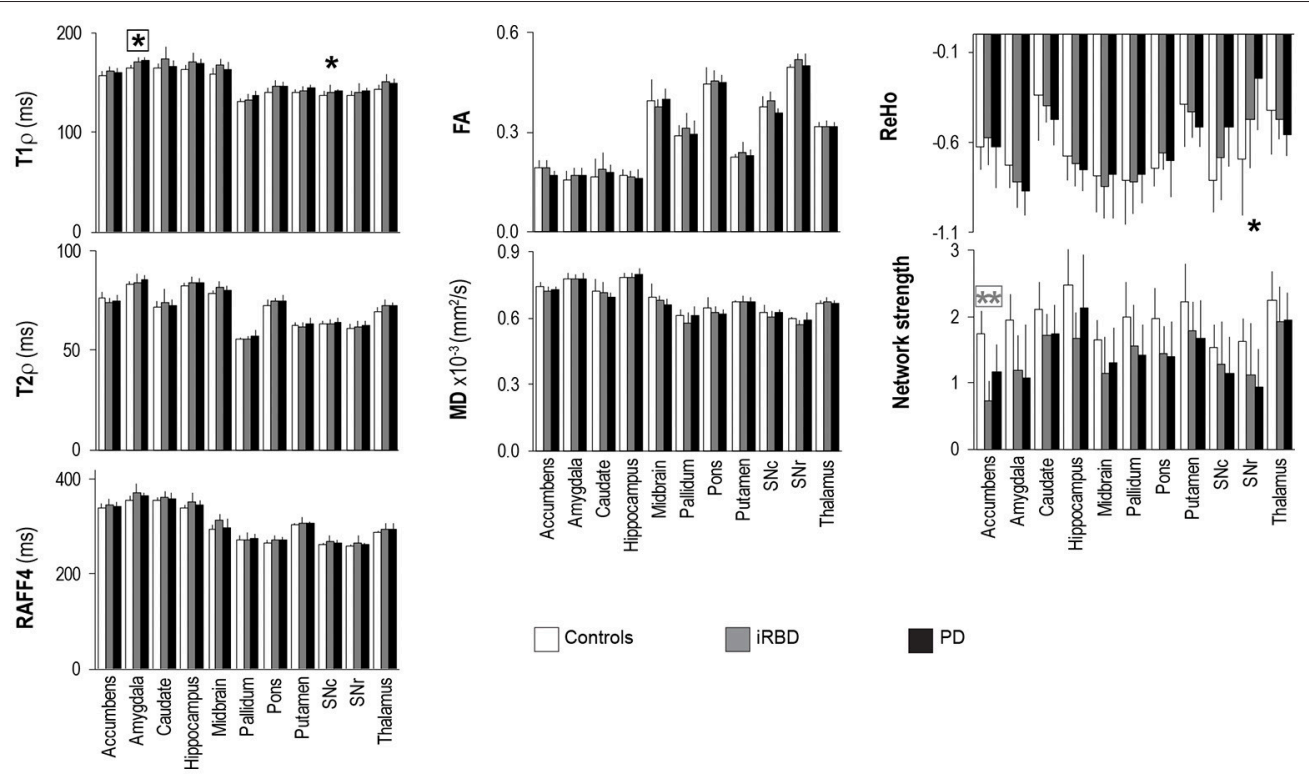

FIGURE 4 | Group summaries of $T_{1}, T_{2 \rho}$, RAFF4, FA, MD, ReHo and network strength. $N=10,8,9$ in the control, iRBD and PD groups, respectively, for $T_{1}, T_{2 \rho}$, and RAFF4, whereas $N=9,8,9$ for FA and MD, and $N=10,8,8$ for network strength and ReHo. Data shown as mean \pm SD. ${ }^{*}$ and ${ }^{* *}$ indicate, respectively, $p<$ 0.05 and $p<0.005$ with age-adjustments after Holm's correction (gray: iRBD vs. controls; black: PD vs. controls). Asterisks within a box indicate $p<0.05$ after correcting FDR for multiple testing (7 modalities). 